\title{
A highly flexible transparent conductive electrode based on nanomaterials
}

\author{
Chang-Lae Kim ${ }^{1}$, Chan-Won Jung ${ }^{2}$, Young-Jei $\mathrm{Oh}^{2}$ and Dae-Eun Kim ${ }^{1}$
}

The electrical, optical, thermal, chemical, mechanical and tribological characteristics of a highly flexible transparent conductive electrode (HFTCE) coating based on reduced graphene oxide ( $\mathrm{GGO}$ ), carbon nanotubes (CNTs) and silver nanowires (AgNWs) were investigated under various conditions. The motivation was to develop a highly durable and flexible film for transparent conductive electrode applications. The overall characteristics of multilayers based on rGO, CNTs and AgNWs were found to be much better than those of the single-layer AgNW coating. The rGO and CNT layers served to protect the AgNW layer from damage due to bending and contact sliding motions. The contact pressure and bending stress were effectively distributed by the CNT layer deposited on top of the AgNW layer due to its spring-like behavior. In addition, the shear force from the friction force was reduced by the rGO top layer, which acted as a solid lubricant. Furthermore, the excellent performance of an HFTCE heater based on the rGO/CNT/AgNW coating was demonstrated by the results of a defrosting test.

NPG Asia Materials (2017) 9, e438; doi:10.1038/am.2017.177; published online 13 October 2017

\section{INTRODUCTION}

In recent decades, various transparent conductive films electrodes have been widely used in electronic devices such as solar cells, displays, memories and batteries. ${ }^{1}$ In particular, indium tin oxide (ITO) has attracted considerable attention as a transparent conductive electrode because of its excellent optical and electrical properties. ${ }^{2}$ However, indium, which is the main raw material used in ITO, has some disadvantages in terms of production and cost. Moreover, ITO application in flexible devices is limited by its brittleness. ${ }^{3}$

To replace ITO in flexible devices, several types of conductive polymers, such as poly(3,4-ethylenedioxythiophene):poly(styrenesulfonate) and poly(3-hexylthiophene-2,5-diyl):[6,6]-phenyl-C61-butyric acid methyl ester have been suggested. ${ }^{4,5}$ However, the electrical efficiency of these conductive polymers is much lower than that of ITO. Moreover, the mechanical properties of these conductive polymers are very weak despite their flexibility. Therefore, it is necessary to combine these conductive polymers with other electrodes to improve efficiency.

Many studies using other types of conductive materials, such as graphene and carbon nanotubes (CNTs), have been conducted. ${ }^{6-12}$ While carbon-based nanosheets and nanowires are known as materials with excellent mechanical and electrical properties, the sheet resistance of the coatings based on carbon is higher. Li et al. ${ }^{6}$ demonstrated that the number of graphene layers deposited on the surface had a significant effect on their conductivity and transmittance. They found that the transmittance decreased, whereas the conductivity increased with the increase in the number of graphene layers. In a study of CNTs as electrodes, Li et al. ${ }^{9}$ observed that CNTs had low conductivity and transmittance. The authors therefore then conducted a study of the combination of graphene and CNTs in which they investigated a composite coating or dual-layer coating with graphene and CNTs. ${ }^{12}$ However, to obtain high conductivity and transmittance, graphene and CNTs should be deposited as one or few layers without any oxidation. The coating process of these types of materials is quite complicated, and they are relatively expensive, so it is difficult to produce them in large quantities. Furthermore, the conductivity values of graphene and CNT layers were much lower than those of ITO and other metal-based electrodes.

Silver nanowires (AgNWs) have been utilized as a transparent conductive electrode in organic photovoltaic devices because of their excellent conductivity and transmittance. ${ }^{13}$ Wang et al. ${ }^{13}$ proved the superiority of AgNWs as electrodes based on properties such as conductivity, transmittance and flexibility. However, the AgNWs deposited on the substrate had a relatively low adhesion force due to the effects of weak van der Waals force and limited contact area between the AgNWs and the substrate. ${ }^{14}$ Thus, it was easy to detach the AgNWs from the substrate by physical/mechanical deformation. Moreover, AgNWs have a low resistance to corrosion because they are composed of a metal and are quite fragile because their dimensions, which are several tens of nanometers in diameter and micrometers in

${ }^{1}$ Center for Nano-Wear, School of Mechanical Engineering, Yonsei University, Seoul, Republic of Korea and ${ }^{2}$ Center for Opto-Electronic Materials, Future Convergence Research Division, Korea Institute of Science and Technology (KIST), Seoul, Republic of Korea

Correspondence: Professor D-E Kim, Center for Nano-Wear, School of Mechanical Engineering, Yonsei University, Yonsei-ro 50, Seodaemun-gu, Seoul 03722, Republic of Korea. E-mail: kimde@yonsei.ac.kr

or Professor Y-J Oh, Center for Opto-Electronic Materials, Future Convergence Research Division, Korea Institute of Science and Technology (KIST), Hwarangno 14-gil 5, Seongbuk-gu, Seoul 02792, Republic of Korea.

E-mail: youngjei@kist.re.kr

Received 4 May 2017; revised 6 July 2017; accepted 30 July 2017 
length. To overcome this problem, a method to strongly fix AgNWs was developed by Gaynor et al. ${ }^{15}$ They embedded AgNWs in a polymer surface, thereby enhancing the adhesion between AgNWs and the substrate because the AgNWs were embedded in the substrate. However, the ultimate mechanical/chemical durability of the AgNW coating was not improved. Ahn et al. ${ }^{16,17}$ improved electrodes based on AgNWs by combining them with graphene. In particular, reduced graphene oxide (rGO) could prevent chemical reactions and thermal oxidation of AgNWs at high temperatures and humidity. In addition, CNTs were used as a protective layer, preventing AgNW oxidation and detachment from the surface upon bending. ${ }^{18,19}$

Accordingly, various materials based on carbon and metal composites have been investigated for use as electrodes by focusing on the electrical and optical properties as well as on their flexibility. By contrast, few studies have investigated the mechanical properties of electrodes. Most studies have focused on the electrical conductivity and/or efficiency of electrodes along with simple bending and stretching tests. ${ }^{20,21}$ Electrode durability has been evaluated using bending and stretching tests with variables such as bending cycle, radius and direction, but there have been far fewer studies of the quantitative mechanical properties of electrodes. In particular, there has been almost no attempt to investigate the relationship between conductivity, transparency, flexibility and mechanical properties.

In this work, the electrical resistance, optical transmittance, thermal properties, mechanical flexibility/durability and tribological characteristics of highly flexible transparent conductive electrodes (HFTCEs) based on multilayer coatings with rGO, multi-walled CNTs and AgNWs were analyzed. Using rGO, CNT and AgNW suspensions, each layer of the multilayer coating was deposited using a spraying method. To protect the AgNW coating that is the main electrode, different types of carbon-based solutions, such as rGO and CNTs, were deposited on the AgNW layer. By controlling the coating method, we were able to optimize the physical properties as well as the optical and electrical properties. The coating structure was varied to improve the mechanical and electrical properties of HFTCEs based on AgNWs. Specifically, single-layer, dual-layer and three-layer coatings based on rGO, CNTs and AgNWs, such as AgNW, rGO/AgNW, CNT/AgNW, AgNW/CNT, rGO/AgNW/CNT and $\mathrm{rGO} / \mathrm{CNT} / \mathrm{AgNW}$, were prepared. The coating layer listed first is on top and the last one listed is at the bottom. The coating conditions for flexible and durable HFTCEs were optimized. The objective of a multilayer coating is to improve the resistance to deformation by physical variations, such as bending, folding and contact scratching, as well as chemical reactions, such as oxidation and corrosion. The quantitative mechanical properties of the coatings were also assessed using micro-tensile, nanoindentation and nano-scratch tests. ${ }^{22-24}$

\section{MATERIALS AND METHODS}

\section{Materials}

The AgNW solution was prepared using our own method based on the polyol process. ${ }^{13,25,26}$ Commercially available rGO and multi-walled CNT solutions were purchased. rGO, CNTs and AgNWs were deposited on polyethylene terephthalate (PET) substrates by spraying.

\section{Experiments}

The surface morphologies before and after the mechanical/tribological tests and the overall and microstructures of all the coatings were visualized by using a three-dimensional (3D) laser scanning confocal microscope (VK-X200, Keyence Co., Osaka, Japan), a field emission scanning electron microscope (JSM-7800F, Jeol Inc., Tokyo, Japan) and a spherical aberration correction scanning transmission electron microscope (JEM-ARM 200F, Jeol Inc., Tokyo,
Japan). The surface roughness and water contact angle of the coatings were measured using a 3D profiler (Dektak XT, Bruker Inc., Tucson, AZ, USA) and a microscope camera (AD4113T, Dino-Lite, Co., Seoul, Republic of Korea), respectively. The mechanical properties of the coatings, such as hardness and elastic modulus, were analyzed by the nano-indentation method (Ultra nanoindentation hardness tester, Anton Paar GmbH Co., Peseux, Switzerland). The tensile tests of the coatings deposited on the PET were performed using a micro-tensile tester (E3000LT, Instron Co., Buckinghamshire, UK). The sheet resistance, transmittance and haze of the coatings were measured using a fourpoint probe system (Keithley 2001 digital multimeter, Keithley Co., Leeds, UK), an ultraviolet/visible spectrophotometer (V-650, JASCO Co., Tokyo, Japan) and a haze spectrophotometer (Haze, Scinco Co., Seoul, Republic of Korea), respectively. The temperature variation of the coating according to the applied voltage was obtained by a thermal imaging camera (TE-P, IR TECH Co., Mahape, India). To assess the friction and wear characteristics of the coatings, the contact ball-on-flat sliding tests were conducted using a custom-built reciprocating-type tribometer. The chemical composition was analyzed by X-ray photoelectron spectroscopy (K-alpha, Thermo Scientific Inc., Warriewood, Australia). Raman spectra were obtained using a Raman spectrometer (DXR Raman microscope, Thermo Scientific Inc., Waltham, MA, USA). The X-ray diffraction (XRD) peaks were measured using a high resolution X-ray diffractometer (SmartLab, Rigaku Co., Tokyo, Japan). The scratch resistance of the coatings was investigated by using a scratch tester (Nanoscratch tester, Anton Paar GmbH Co., Peseux, Switzerland).

\section{RESULTS AND DISCUSSION}

The physical surface morphology and structure of each coating were observed using a 3D laser scanning confocal microscope and a field emission scanning electron microscope, respectively, as shown in Figure 1a and c. It was confirmed that each coating had a distinct surface morphology, as shown in the $3 \mathrm{D}$ laser scanning confocal microscope images of Figure 1a. Field emission scanning electron microscope images of the surface and cross-sections of a single-layer AgNW coating and three-layer coatings based on rGO, CNTs and AgNWs are shown in Figure 1c. The morphology and the structure of the coatings can be clearly observed. The surface roughness measurements of the coatings made by a 3D profiler were compared, as shown in Figure $1 \mathrm{~b}$. The value of the surface roughness increased with the deposition of CNT layers and decreased with the deposition of an rGO layer. It was supposed that the two-dimensional sheets of rGO induced a smoother surface, while the $3 \mathrm{D}$ structure of the CNTs caused the coating surface to become rougher. Furthermore, the surface energy of the coatings was investigated by measuring the water contact angle. As shown in the inset images of Figure 1b, the water contact angles of AgNW, rGO/AgNW, CNT/AgNW, AgNW/CNT, rGO/AgNW/CNT and $\mathrm{rGO} / \mathrm{CNT} / \mathrm{AgNW}$ coatings were measured as $54^{\circ}, 88^{\circ}, 81^{\circ}, 77^{\circ}$, $84^{\circ}$ and $92^{\circ}$, respectively. These results indicated that the surface energy of the AgNW coating was higher than those of other coatings formed with two or three layers based on rGO and CNTs.

The chemical compositions of the coatings were investigated by $\mathrm{X}$-ray photoelectron spectroscopy (Supplementary Figure S1). The $\mathrm{X}$-ray photoelectron spectroscopy data show that the AgNW coating consists of Ag, $\mathrm{O}$ and $\mathrm{C}$, while both the rGO and CNT coatings contain $\mathrm{C}, \mathrm{O}$ and N. Moreover, each coating shows several highintensity peaks at their intrinsic ranges of binding energy. The crystal structure, disorder and defects of the rGO, CNT and rGO/CNT coatings were assessed by Raman spectroscopy (Supplementary Figure S2). The microstructures of each layer were characterized by XRD (Supplementary Figure S3). The interlayer spacing can be calculated using XRD.

More details and further evidence of the structural characteristics of the $\mathrm{rGO} / \mathrm{CNT} / \mathrm{AgNW}$ coating were investigated using transmission electron microscopy (TEM). Figure 1d shows the TEM image of the 
cross-section of the rGO/CNT/AgNW coating at low magnification. The arrangement of atoms as well as the interface of each layer is observed at a higher magnification, as shown in Figure 1e. In the selected area electron diffraction pattern of the $\mathrm{rGO} / \mathrm{CNT} / \mathrm{AgNW}$ coating (Supplementary Figure S4), the diffraction rings indicating the face-centered cubic structure of Ag that was determined using XRD were observed. Supplementary Figure S5 shows the high-magnification TEM images of the rGO, CNT and AgNW layers. Using these TEM images, the atomic lattice structure of each layer can be clearly confirmed. It can be proven that the value of the interlayer spacing measured by the TEM images at high magnification corresponds with that calculated by the XRD analysis. In addition, in the TEM image of the rGO layer, it can be confirmed that the number of rGO layers is $\sim 30$.

The mechanical properties of the coatings, such as hardness and elastic modulus, were analyzed by performing nano-indentation tests, as shown in Figure 2a. The mechanical properties of the $\mathrm{rGO} / \mathrm{AgNW}$ coating improved significantly compared to the AgNW coating. Moreover, the values of the hardness and elastic modulus of the $\mathrm{rGO} / \mathrm{AgNW} / \mathrm{CNT}$ and $\mathrm{rGO} / \mathrm{CNT} / \mathrm{AgNW}$ coatings greatly increased compared to the AgNW/CNT and CNT/AgNW coatings, respectively. This improvement in the mechanical properties indicates that the mechanical properties of the coatings were greatly influenced by rGO at the top layer. However, the hardness and elastic modulus of the AgNW/CNT and CNT/AgNW coatings decreased compared to the AgNW coating, except for the elastic modulus of the CNT/AgNW coating, which increased slightly. Furthermore, there was a significant reduction in the values of both the hardness and elastic modulus of the $\mathrm{rGO} / \mathrm{AgNW} / \mathrm{CNT}$ coating compared to those of the rGO/AgNW coating. This outcome was attributable to the morphological variation of the coating in the presence of CNTs, which had a mesh-type structure. In other words, the coating with $\mathrm{rGO} / \mathrm{AgNW}$ layers deposited on a CNT layer with a mesh structure resulted in lower mechanical properties compared to the coating with rGO/AgNW layers deposited on a flat substrate without the CNT layer. Essentially, the CNT layer with a mesh structure provided added compliance to the coating layers, thus causing a decrease in both hardness and elastic modulus.

The tensile stress variations with the tensile strain of all the coatings were measured by micro-tensile tests, as shown in Figure $2 \mathrm{~b}$. It was also confirmed that the mechanical properties of the coatings were affected much more by the rGO layer than the CNT layer. From the slopes of the stress-strain curves, the force at 5\% strain and tensile strain at the maximum force applied in the tensile test can be obtained, as shown in Supplementary Figure S6. The variation trend of the force at $5 \%$ strain almost corresponds with that of the mechanical properties analyzed by nano-indentation.

The electrical and optical properties of the coatings for a transparent conductive electrode were assessed, as shown in Figure $2 \mathrm{c}$ and $\mathrm{d}$. The sheet resistance and transmittance of the multilayer coatings based on rGO, CNTs and AgNWs decreased by $\sim 30 \%$ and $~ 10 \%$, respectively, compared to those ( $75 \Omega$ per square and 95\%) of the AgNW coating. In other words, the electrical conductivity of the coatings improved, while the transparency decreased slightly. In the case of coating reflectance, both the rGO and CNT layers showed a reduction in the haze of $20-55 \%$ compared to the single layer of AgNWs (Figure 2d).

The thermal properties of the coatings based on rGO, CNTs and AgNWs were compared according to the applied direct current voltage, as shown in Figure 2e. At the same direct current voltage, the coatings with the protective layers showed higher temperature a
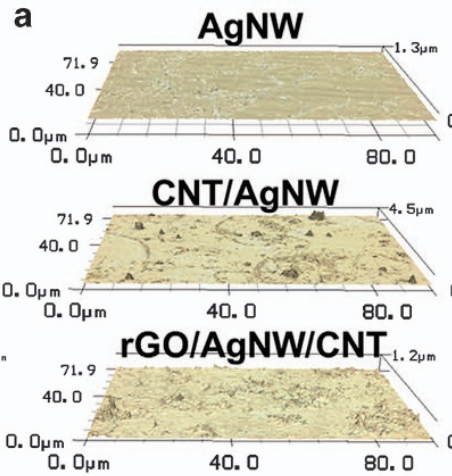
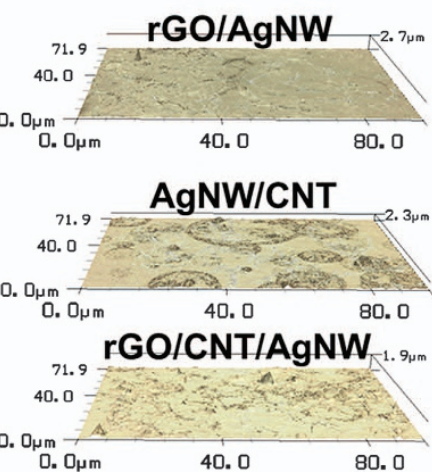
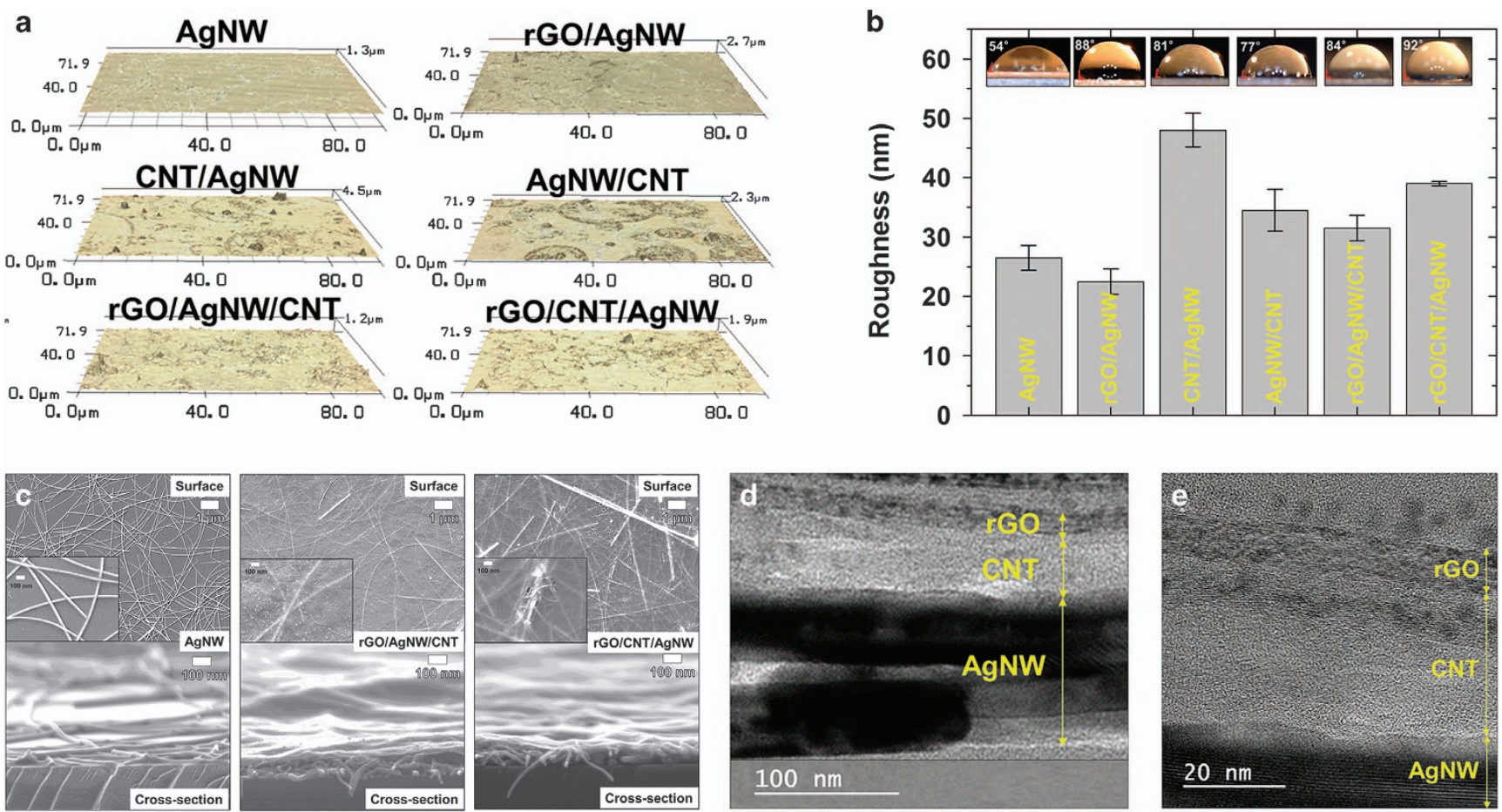

Figure 1 Physical and surface properties of the coatings based on reduced graphene oxide (rGO), carbon nanotubes (CNTs) and silver nanowires (AgNWs). (a) Three-dimensional (3D) laser scanning confocal microscope images of the surface morphologies. (b) Surface roughness measured using a 3D profiler (inset images: contact angles of the coatings). (c) Scanning electron microscopy images of the surfaces and the cross-sections of the coatings. (d, e) Transmission electron microscopy image of the cross-section of the rGO/CNT/AgNW coating at (d) low and (e) high magnification. 
compared to the single layer of AgNWs. Moreover, the heating effect of the coatings formed with rGO and CNT layers together was slightly better than that of dual-layer coatings where the protective layer was used separately. The infrared images inset in the voltage-temperature $(V-T)$ graph of Figure 2e show the temperature variation of $\mathrm{rGO} /$ $\mathrm{CNT/AgNW}$ coating step by step with increasing input voltage. Using the $V-T$ data, the temperature variation at the steady state as a function of areal power density (the power applied by Joule heating per unit area ${ }^{27}$ for all the coatings based on the AgNW layer with different combinations of protective layers, such as rGO and CNTs, can be compared, as shown in Supplementary Figure S7.

The durability assessments with respect to the aging time of the coatings based on rGO, CNTs and AgNWs were performed by measuring the variation of sheet resistance, as shown in Figure 3a.
The rGO/CNT/AgNW coating showed the best durability in that the variation $\left(R-R_{0}\right) / R_{0}$ of sheet resistance was the smallest among all the coatings during over $2500 \mathrm{~h}$ under the atmospheric conditions in a certain range of temperature $\left(17-32^{\circ} \mathrm{C}\right)$ and humidity $(25-65 \%)$. Overall, rGO and CNT layers played important roles as protective layers to maintain the conductivity of the AgNWs when they were deposited on the AgNW layer, while the electrical properties of the coatings with the CNT layer at the bottom were not stable over the long aging time. In general, the coatings with rGO and CNT layers showed superior durability over the aging time compared to the single layer of AgNWs, except the AgNW/CNT coating. It was supposed that top layers of rGO and CNTs could protect the AgNWs against corrosion and oxidation due to the variation of temperature and humidity. ${ }^{16-19}$

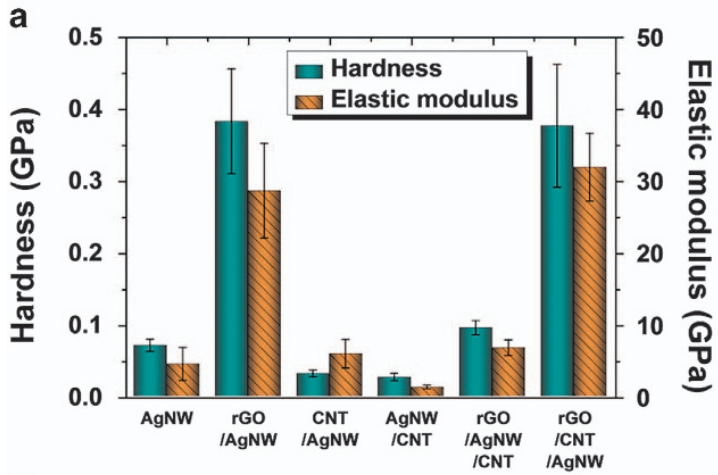

b
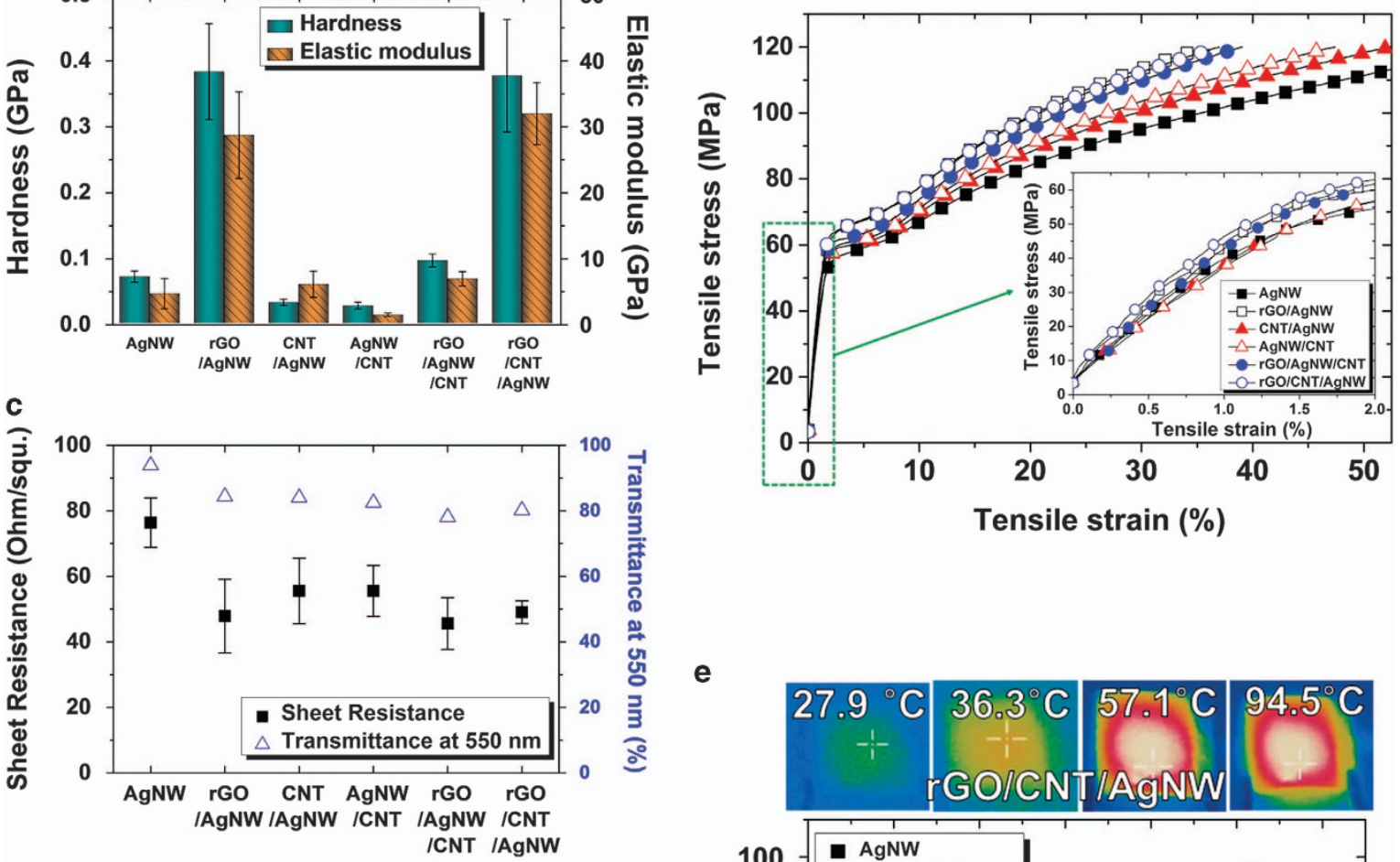

e
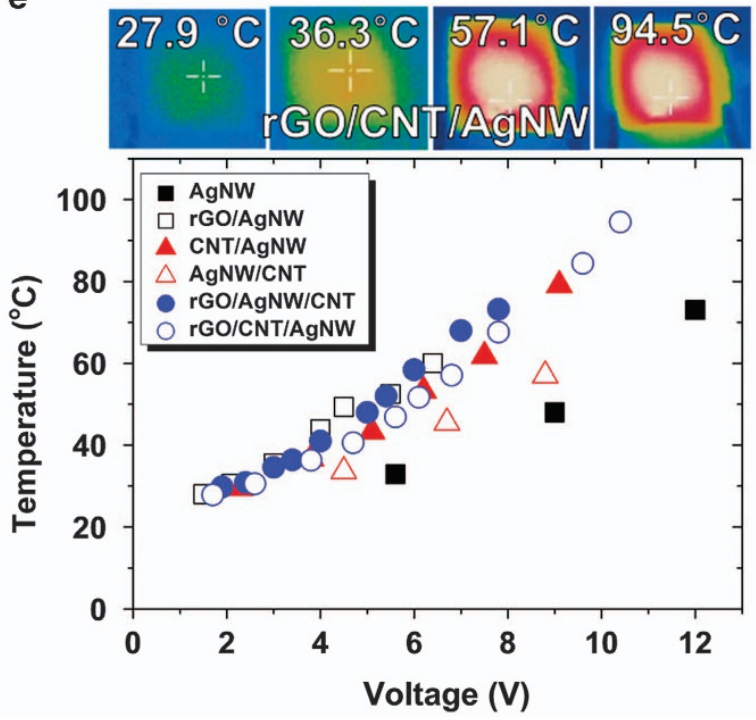

Figure 2 Mechanical, electrical, optical and thermal properties of the coatings based on reduced graphene oxide (rGO), carbon nanotubes (CNTs) and silver nanowires (AgNWs). (a) Hardness and elastic modulus values of the coatings measured by nano-indentation. (b) Tensile stress-strain curves (TSSCs) of the coatings measured using micro-tensile tests, including the inset TSSC graph for $2 \%$ strain. (c) Average sheet resistance and transmittance at $550 \mathrm{~nm}$. (d) Haze over the visible range. (e) Temperature variation with respect to the applied voltage (top: thermal images of temperature variation of the rGO/CNT/ AgNW coating). 
The durability of the flexibility of the coatings according to the number of folding cycles was investigated by measuring $\left(R-R_{0}\right) / R_{0}$, as shown in Figure 3b. The experimental results showed that not only the material combination but also the layer stacking sequence affected the performance of the flexible conductive electrode. By combining Ag nanowires with both rGO and CNT layers, superior bending properties with good durability could be achieved. The $\left(R-R_{0}\right) / R_{0}$ of the $\mathrm{rGO} / \mathrm{CNT} / \mathrm{AgNW}$ coating was the lowest among all the coatings after completion of the folding test (2000 cycles). Such an outcome could be attributed to the synergistic effects of the coating layers or materials. In the folding test, the CNT coating on top of the AgNWs acted as a protective layer that could prevent AgNW detachment by physical deformation. In addition, the rGO deposited on the CNT layer could prevent plastic deformation of the CNT structure against a large range of tensile and compressive strains. In general, whereas the rGO and the CNT layers could protect the conductivity of the coating when they were deposited on the AgNW layer, the $\left(R-R_{0}\right) / R_{0}$ of the coatings with the CNT layer at the bottom, such as the AgNW/CNT and $\mathrm{rGO} / \mathrm{AgNW} / \mathrm{CNT}$ coatings, significantly increased after a relatively small number of folding cycles compared to the single layer of AgNWs. In other words, the structure with the CNT on the bottom layer was not suitable for maintaining the AgNWs at the top. It should be noted that the position of the protective layers of rGO and CNTs has a considerable effect on the durability and flexibility of the coatings. In addition, the durability and reliability as well as the fundamental concepts and characteristics of the coatings based on AgNW as a heater were compared between the present and previous studies (Table 1). ${ }^{28-34}$ In summary, given the good electric conductivity of AgNWs, deposition of CNTs and rGO sequentially on the AgNWs provided excellent bending durability. This clearly demonstrated that there was a synergistic effect of combining rGO with CNTs in improving the bending durability.

The thermal and chemical resistance of the coatings were compared when the coatings were put in the oven at $100^{\circ} \mathrm{C}$ and immersed in different types of solutions, such as deionized water, ethanol, isopropanol and acetone for $15 \mathrm{~h}$ (Supplementary Figure S8). It was confirmed that the thermal and chemical durability of the AgNW/ CNT coating was weakest among all the coatings.

The friction characteristics of all the coatings were investigated by comparing the coefficient of friction (COF) according to the total number of sliding cycles (Supplementary Figure S9). The average COF values of each coating with respect to the initial and final stages can be clearly compared, as shown in Figure 3c. From the decrease in the average COF of the rGO/AgNW coating compared to the AgNW coating, it can be noted that rGO acted as a lubricant layer for AgNWs against the contact sliding motion. Moreover, the rGO layer was an excellent lubricant in the $\mathrm{rGO} / \mathrm{AgNW} / \mathrm{CNT}$ and the $\mathrm{rGO} / \mathrm{CNT} / \mathrm{AgNW}$ coatings compared to the AgNW/CNT and the CNT/AgNW coatings. However, the CNT layer showed different trends of the average COF according to the layer position. It was supposed that the increase in contact area and surface roughness associated with the CNT layer led to an increase in the average COF. From these results, it can be stated that the layer position is important in determining the friction characteristics of the coating. Despite the fact that rGO was the top a

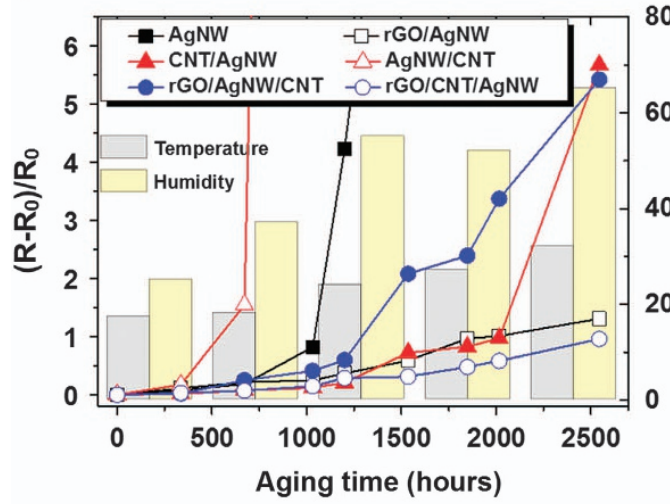

b

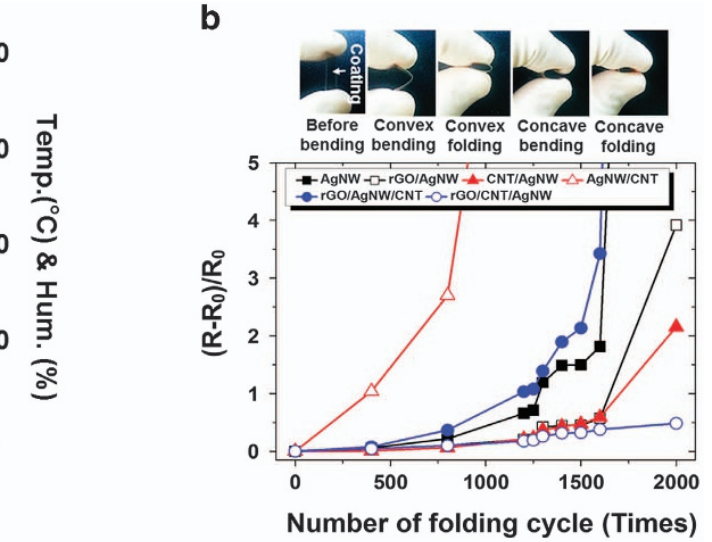

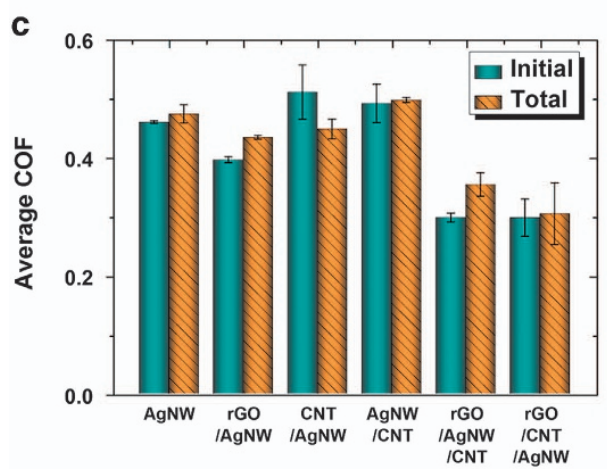

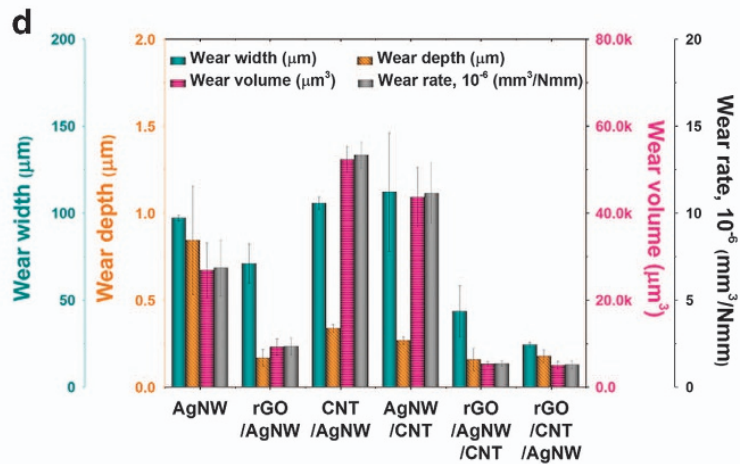

Figure 3 Electrical/mechanical durability, friction and wear characteristics of the coatings based on reduced graphene oxide (rGO), carbon nanotubes (CNTs) and silver nanowires (AgNWs). (a) Variation rate of sheet resistance according to the aging time, temperature and humidity. (b) Variation rate of sheet resistance according to the number of folding cycles (top: images of bending and folding motions with different convex and concave directions). (c) Average coefficient of friction (COF) at the initial and final stages measured by the contact sliding tests. (d) Wear width, depth, volume and rate formed after the contact sliding tests. 
layer for both $\mathrm{rGO} / \mathrm{AgNW}$ and $\mathrm{rGO} / \mathrm{CNT} / \mathrm{AgNW}$ coatings, the latter coating showed lower friction because of the presence of the CNT layer in between the top rGO layer and the bottom AgNW layer. Overall, the average COF values of the $\mathrm{rGO} / \mathrm{CNT} / \mathrm{AgNW}$ coating at the initial and final stages were the lowest among those of all the coatings, which showed a decrease of $35 \%$ and $36 \%$, respectively, compared to those of the AgNW coating. However, it should be mentioned that the average COF of the specimens with the rGO layer was in the range of $0.3-0.4$, which was higher than those of the graphene or the rGO coatings $(0.1-0.25)$ reported in previous studies. ${ }^{35-38}$ The higher COF was attributed to the fact that the $\mathrm{CNT}$ and AgNW layers below the rGO layer caused the surface roughness of the coating to increase, which in turn led to the increase in $\mathrm{COF}$ compared with smooth graphene or rGO coatings without such underlayers.

The wear characteristics of the coatings obtained using the contact sliding test were compared with the values of wear width, depth, volume and rate, as shown in Figure 3d. As shown in the figure, the wear of the $\mathrm{rGO} / \mathrm{CNT} / \mathrm{AgNW}$ coating was lower than those of the other coatings. Specifically, the wear width, depth, volume and rate of the $\mathrm{rGO} / \mathrm{CNT} / \mathrm{AgNW}$ coating were $~ 75 \%, 79 \%, 82 \%$ and $82 \%$ lower, respectively, than those of the AgNW coating. The wear values of the $\mathrm{rGO} / \mathrm{AgNW}$ coating significantly decreased compared to those of the AgNW coating. It can be proven that rGO acted as an outstanding protective layer for AgNWs with respect to the contact sliding action. Furthermore, it was shown that $\mathrm{rGO}$ at the top layer of the $\mathrm{rGO} /$ AgNW/CNT and the $\mathrm{rGO} / \mathrm{CNT} / \mathrm{AgNW}$ coatings acted as a protective layer for the AgNW/CNT and the CNT/AgNW coatings. On the other hand, from the results, it was supposed that the CNT layer with the flexible mesh-type structure could prevent penetration of the coating, although it was easily compressed so that the contact area between the tip and the coating surface increased. From the fact that the amount of wear of the $\mathrm{rGO} / \mathrm{CNT} / \mathrm{AgNW}$ coating was lower than those of the $\mathrm{rGO} / \mathrm{AgNW}$ and the CNT/AgNW coatings, it was apparent that the CNT layer in between the top rGO layer and the bottom AgNW layer played a critical role in reducing the wear. Essentially, the CNT layer provided the spring effect, which was helpful in distributing the contact force over a larger area. Furthermore, the top rGO layer allowed the surface shear stress to be lowered due to its relatively low frictional property. Moreover, it was presumed that the film formed on the ball surface that was transferred from the CNT layer aided in decreasing the wear of the coating surface (Supplementary Figure S10). ${ }^{39}$

To assess the scratch durability of the HFTCE coating based on rGO, CNTs and AgNWs, nano-scratch tests were conducted (Supplementary Figure S1la and b). According to the scratch length, the COF values of the $\mathrm{rGO} / \mathrm{AgNW} / \mathrm{CNT}$ and the $\mathrm{rGO} / \mathrm{CNT} / \mathrm{AgNW}$ coatings were much lower than those of other coatings (Supplementary Figure S11a). This result was quite similar to that of the sliding tests performed with a ball tip under a load of $9.81 \mathrm{mN}$ for 100 cycles. The critical load as well as the friction force and the total average value of the COF with respect to all the coatings were compared (Supplementary Figure S11b). Among all the coatings, the critical load for the $\mathrm{rGO} / \mathrm{CNT} / \mathrm{AgNW}$ coating was the highest, which increased $\sim 83 \%$ compared to the AgNW coating. In contrast, the values of the friction force and COF decreased $\sim 29 \%$ and $61 \%$, respectively.

The durability and flexibility of the HFTCE coatings can be assessed from the damage images of the surface of all the coatings after the folding tests shown in Figure 4a. Many detached parts with large cracks were generated on the surfaces of the AgNW, rGO/AgNW, 
a

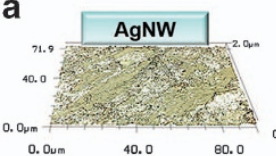

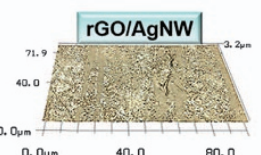
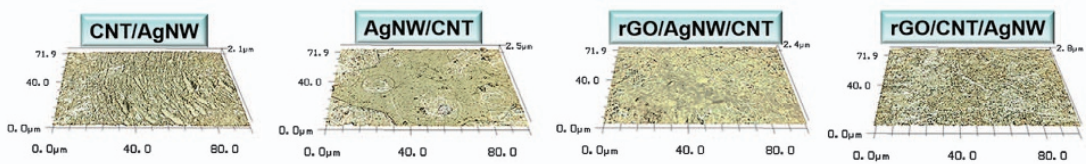

b
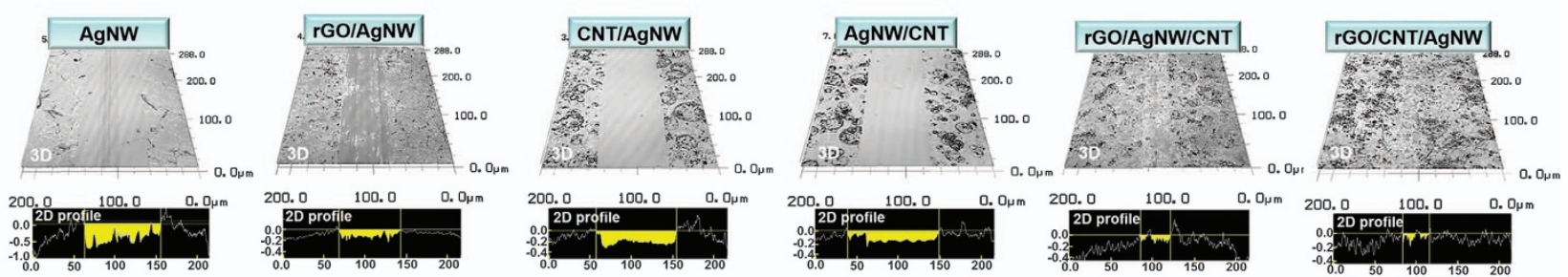

C
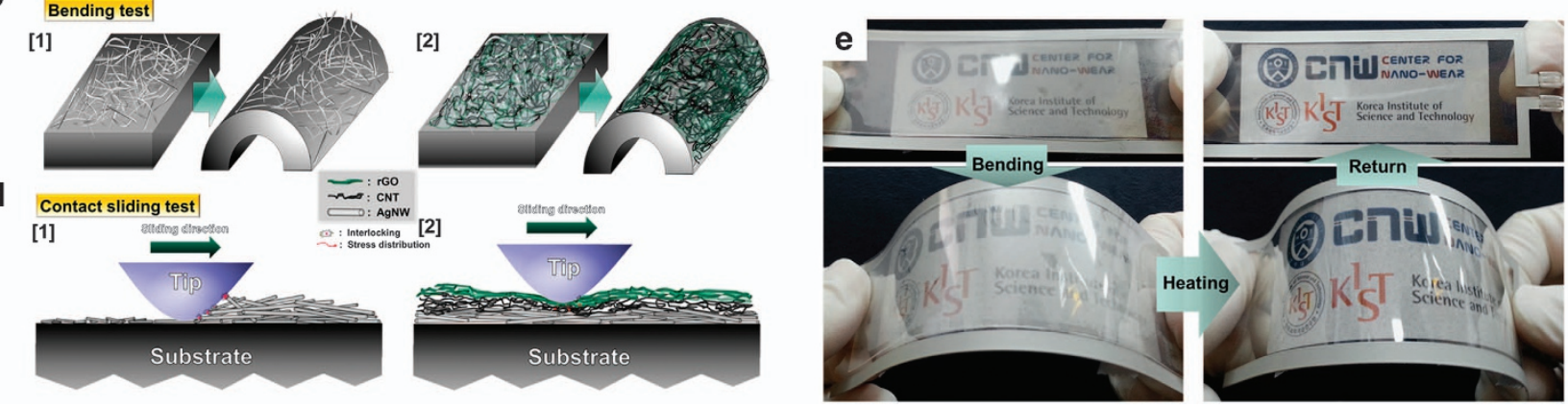

Figure 4 Durability upon bending/folding and sliding motions of the coatings based on reduced graphene oxide (rGO), carbon nanotubes (CNTs) and silver nanowires (AgNWs). (a, b) Three-dimensional laser scanning confocal microscope images of (a) surface damage after the folding tests and (b) wear tracks after the contact sliding tests (bottom: two-dimensional (2D) profile images of the wear tracks. (c, d) Schematic designs comparing the damage/wear mechanism of $(\mathbf{c}$ ) bending and (d) contact sliding motions between the AgNW and the rGO/CNT/AgNW coatings. (e) Defrosting test of the completed unit of the HFTCE heater based on the rGO/CNT/AgNW coating, confirming the durability of the flexibility.

CNT/AgNW, AgNW/CNT and rGO/AgNW/CNT coatings, while only a small crack was found on the surface of the $\mathrm{rGO} / \mathrm{CNT} / \mathrm{AgNW}$ coating. The reason that the $\left(R-R_{0}\right) / R_{0}$ of the AgNW/CNT coating markedly increased (Figure $3 \mathrm{~b}$ ) can be easily explained by the damage after the folding test. It should be noted that the AgNWs detached over a large area so that there was disconnect between the nanowires. On the other hand, rGO/CNT/AgNW can be utilized as an HFTCE for flexile devices that demand mechanical stability and flexibility.

The wear tracks on the coating surfaces were compared, as shown in Figure 4b. The AgNW coating was completely removed after the sliding test, as shown in the $3 \mathrm{D}$ confocal image. The substrate (PET) was penetrated because the penetration depth observed in the twodimensional profile of the wear track is larger than the thickness of the AgNW coating. On the other hand, it was confirmed that the coatings with the CNT layer could protect the substrate. It is supposed that the contact force and shear force generated during the sliding test might be distributed through stiffness control of the CNT layer, which is called the spring effect. ${ }^{39,40}$ It was also determined that rGO acted as a protective layer on the AgNWs as there was almost no wear track on the surfaces of either the $\mathrm{rGO} / \mathrm{AgNW} / \mathrm{CNT}$ or the $\mathrm{rGO} / \mathrm{CNT} / \mathrm{AgNW}$ coatings. It should be noted that the rGO and the CNT layers were effective in protecting the AgNW layer when they were applied together, whereas a layer of rGO or CNTs alone was not enough to protect the AgNW layer. Overall, the multilayer coating comprised of $\mathrm{rGO} / \mathrm{CNT} / \mathrm{AgNW}$ layers showed the best wear resistance against contact sliding.

From the analysis of the damage phenomena of the surfaces of all the coatings after the folding and contact sliding tests mentioned above, the main damage mechanism may be described by the schematics shown in Figure $4 \mathrm{c}$ and d. Figure $4 \mathrm{c}(1)$ and (2) shows the variations in the surface morphologies of the AgNW and the rGO/ $\mathrm{CNT} / \mathrm{AgNW}$ coatings upon bending, respectively. It was presumed that the network of AgNWs was disrupted around the bent/folded area after the repeated bending/folding motions because of the weak van der Waals force and limited contact area between the AgNWs and the substrate. ${ }^{14}$ Finally, nanowires that experienced a relatively large change would be detached from the substrate. On the other hand, in the case of the rGO/CNT/AgNW coating, the CNT layer might keep the AgNWs attached to the substrate and rGO might prevent the CNT layer from deforming with a severe strain. ${ }^{16-19}$ Next, the schematic designs of the wear mechanism of the contact sliding test can be described as shown in Figure $4 \mathrm{~d}(1)$ and (2). Figure $4 \mathrm{~d}(1)$ shows that the AgNW coating is easily removed by the tip-sliding motion. The edges of the nanowires might be able to increase the interlocking with the tip surface against the sliding direction so that it will cause high friction. In the case of the $\mathrm{rGO} / \mathrm{CNT} / \mathrm{AgNW}$ coating (Figure $4 \mathrm{~d}(2)$ ), rGO on the top can protect the bottom layers because of its high durability and lead to slipping by means of a low shear force. Furthermore, the CNTs under the rGO layer can distribute the contact force and shear force generated on the upper layer by means of the spring effect. ${ }^{39,40}$

On the basis of the experimental results, it may be stated that the friction and wear characteristics of the $\mathrm{rGO} / \mathrm{CNT} / \mathrm{AgNW}$ coating are the best among all the coatings. Such an outcome was due to the synergistic effect of combining rGO with CNTs on top of the AgNW layer. The mechanism of this synergistic effect could be described by the spring effect of the CNT layer with a mesh structure that deformed easily, which allowed the contact and shear forces to be distributed 
over a larger area. Distribution of the forces over a larger area led to a decrease in the stresses built up in the coating, which was beneficial in reducing friction and wear. Furthermore, if only CNTs were deposited on the AgNWs, the spring effect was not effective because of the high friction caused by the interlocking of the CNT fibers with the counter surface. Thus, for the spring effect to be effective, the high friction caused by the interlocking phenomenon of the CNT fibers needed to be suppressed. This could be achieved by depositing the rGO layer, which has a relatively low friction, on top of the CNT layer. In summary, the benefits that could be attained by depositing rGO and CNT layers on top of AgNWs could only be derived by the synergistic effects of these materials.

To prove the superiority of the concept of coating proposed in this work, it was applied to an actual device. We prepared the complete unit of a flexible transparent heater, as shown in Supplementary Figure S12 and Figure 4e. The durability and flexibility of the heater, which was an HFTCE based on the rGO/CNT/AgNW coating, were assessed by heating and bending tests. First, a frosted cover glass $(75 \mathrm{~mm} \times 25 \mathrm{~mm})$ was placed on the heater $(120 \mathrm{~mm} \times 40 \mathrm{~mm})$ based on the rGO/CNT/AgNW coating, as shown in Supplementary Figure S12. Frost on the cover glass was formed in the freezer at $-20^{\circ} \mathrm{C}$ for $24 \mathrm{~h}$. After applying a voltage to increase the temperature to $\sim 50{ }^{\circ} \mathrm{C}$, the frost on the cover glass was clearly removed. Next, a frosted PET plate was placed on the heater so that the logos and letters on the back side of the heater were not clearly seen. The HFTCE heater was bent by applying the voltage to achieve a temperature of $\sim 50{ }^{\circ} \mathrm{C}$. It was confirmed that the frost was completely removed during the bending motion so that the logos and letters were clearly visible. After the frost was removed, the heater returned to its original shape. These bending and heating tests were repeated more than 10 times; nevertheless, the performance of the heater was still maintained. It is noted that the HFTCE heater based on the $\mathrm{rGO} / \mathrm{CNT} / \mathrm{AgNW}$ coating has superior durability and flexibility with excellent thermal performance.

\section{CONCLUSIONS}

In this study, HFTCE coatings based on rGO, CNTs and AgNWs were developed. The electrical, optical, thermal, chemical, mechanical and tribological characteristics of the HFTCE coatings were assessed under various conditions. It was confirmed that protective layers, such as rGO and CNTs, improved the durability of the electrical conductivity of the AgNW layer. In particular, HFTCE coatings with multilayers based on rGO, CNTs and AgNWs had excellent mechanical, chemical and thermal properties. Moreover, they showed superior flexibility and durability as well as lower friction and wear characteristics compared to a single layer of AgNWs. The main mechanism of damage on the HFTCE coating surface was established using folding and sliding tests. Deformation of the AgNWs by the bending motion was evidently reduced by means of the protective layers, such as rGO and CNTs, which prevented the $\mathrm{CNT}$ and AgNW layers from detaching. Furthermore, the rGO- and CNT-based protective layers prevented damage to the AgNWs from the contact sliding motion, whereas the single-layer AgNW coating was easily removed by the generation of high friction. It is thought that the CNTs in the layer on top of the AgNWs were able to distribute the contact pressure, similar to the effect of a spring with high flexibility. It should be noted that rGO as the top layer reduced the shear force induced by the friction force by acting as a lubricant, providing excellent durability and mechanical properties. The HFTCE coating ( $\mathrm{rGO} / \mathrm{CNT} / \mathrm{AgNW}$ ) was used as a heater, demonstrating the superiority of its performance by frost removal tests on glass and PET substrates.

\section{CONFLICT OF INTEREST}

The authors declare no conflict of interest.

\section{ACKNOWLEDGEMENTS}

This work was supported by the National Research Foundation of Korea (NRF) grant funded by the Korea government (MSIT) (No. 2010-0018289).

\section{PUBLISHER'S NOTE}

Springer Nature remains neutral with regard to jurisdictional claims in published maps and institutional affiliations.

1 Park, J., Shin, K. \& Lee, C. Roll-to-roll coating technology and its applications: a review. Int. J. Precis. Eng. Manuf. 17, 537-550 (2016).

2 Hotovy, J., Hüpkes, J., Böttler, W., Marins, E., Spiess, L., Kups, T., Smirnov, V., Hotovy, I. \& Kováč, J. Sputtered ITO for application in thin-film silicon solar cells: relationship between structural and electrical properties. Appl. Surf. Sci. 269, 81-87 (2013).

3 Yang, C. W. \& Park, J. W. The cohesive crack and buckle delamination resistances of indium tin oxide (ITO) films on polymeric substrates with ductile metal interlayers. Surf. Coat. Technol. 204, 2761-2766 (2010).

4 Kaltenbrunner, M., White, M. S., Głowacki, E. D., Sekitani, T., Someya, T., Sariciftci, N. S. \& Bauer, S. Ultrathin and lightweight organic solar cells with high flexibility. Nat. Commun. 3, 770 (2012).

5 Yagci, Ö., Yesilkaya, S. S., Yüksel, S. A., Ongül, F., Varal, N. M., Kus, M., Günes, S. \& Icelli, O. Effect of boric acid doped PEDOT:PSS layer on the performance of P3HT: PCBM based organic solar cells. Synth. Met. 212, 12-18 (2016).

6 Li, X., Zhu, Y., Cai, W., Borysiak, M., Han, B., Chen, D., Piner, R. D., Colombo, L. \& Ruoff, R. S. Transfer of large-area graphene films for high-performance transparent conductive electrodes. Nano Lett. 9, 4359-4363 (2009).

$7 \mathrm{Kim}$, H. J., Seo, K. J. \& Kim, D. E. Investigation of mechanical behavior of single- and multi-layer graphene by using molecular dynamics simulation. Int. J. Precis. Eng. Manuf. 17, 1693-1701 (2016).

$8 \mathrm{Kim}$, H. J., Shin, D. G. \& Kim, D. E. Frictional behavior between silicon and steel coated with graphene oxide in dry sliding and water lubrication conditions. Int. J. Precis. Eng. Manuf. 3, 91-97 (2016).

9 Li, Z., Kandel, H. R., Dervishi, E., Saini, V., Xu, Y., Biris, A. R., Lupu, D., Salamo, G. J. \& Biris, A. S. Comparative study on different carbon nanotube materials in terms of transparent conductive coatings. Langmuir 24, 2655-2662 (2008).

10 Shin, Y. C., Novin, E. \& Kim, H. Electrical and thermal conductivities of carbon fiber composites with high concentrations of carbon nanotubes. Int. J. Precis. Eng. Manuf. 16, 465-470 (2015).

11 Bae, K. M., Yang, H. D., Tufa, L. T. \& Kang, T. J. Thermobattery based on CNT coated carbon textile and thermoelectric electrolyte. Int. J. Precis. Eng. Manuf. 16, 1245-1250 (2015).

12 Zhu, Y., Li, L., Zhang, C., Casillas, G., Sun, Z., Yan, Z., Ruan, G., Peng, Z., Raji, A.-R. O., Kittrell, C., Hauge, R. H. \& Tour, J. M. A seamless three-dimensional carbon nanotube graphene hybrid material. Nat. Commun. 3, 1225 (2012).

13 Wang, B. Y., Yoo, T. H., Lim, J. W., Sang, B. I., Lim, D. S., Choi, W. K., Hwang, D. K. \& $\mathrm{Oh}, \mathrm{Y}$. J. Enhanced light scattering and trapping effect of Ag nanowire mesh electrode for high efficient flexible organic solar cell. Small 11, 1905-1911 (2015).

$14 \mathrm{Kim}$, H. J., Kang, K. H. \& Kim, D. E. Sliding and rolling frictional behavior of a single $\mathrm{ZnO}$ nanowire during manipulation with an AFM. Nanoscale 5, 6081-6087 (2013).

15 Gaynor, W., Burkhard, G. F., McGehee, M. D. \& Peumans, P. Smooth nanowire/polymer composite transparent electrodes. Adv. Mater. 23, 2905-2910 (2011).

16 Ahn, Y., Jeong, Y. \& Lee, Y. Improved thermal oxidation stability of solution-processable silver nanowire transparent electrode by reduced graphene oxide. ACS Appl. Mater. Interfaces 4, 6410-6414 (2012).

17 Han, J. T., Jeong, B. H., Seo, S. H., Roh, K. C., Kim, S., Choi, S., Woo, J. S., Kim, H. Y., Jang, J. I., Shin, D.-C., Jeong, S., Jeong, H. J., Jeong, S. Y. \& Lee, G.-W. Dispersantfree conducting pastes for flexible and printed nanocarbon electrodes. Nat. Commun. 4 , 2491 (2013).

18 Pillai, S. K. R., Wang, J., Wang, Y., Sk, M. M., Prakoso, A. B., Rusli \& Chan-Park, M. B. Totally embedded hybrid thin films of carbon nanotubes and silver nanowires as flat homogenous flexible transparent conductors. Sci. Rep. 6, 38453 (2016).

19 Kim, D., Zhu, L., Jeong, D.-J., Chun, K., Bang, Y.-Y., Kim, S.-R., Kim, J.-H. \& Oh, S.-K. Transparent flexible heater based on hybrid of carbon nanotubes and silver nanowires. Carbon 63, 530-536 (2013).

20 Liang, J., Li, L., Chen, D., Hajagos, T., Ren, Z., Chou, S.-Y., Hu, W. \& Pei, Q. Intrinsically stretchable and transparent thin-film transistors based on printable silver nanowires, carbon nanotubes and an elastomeric dielectric. Nat. Commun. 6, 7647 (2015).

21 Yoon, S. S. \& Khang, D. Y. Room-temperature chemical welding and sintering of metallic nanostructures by capillary condensation. Nano Lett. 16, 3550-3556 (2016).

22 Jeong, I., Kim, H. J., Lee, B. S., Son, H. J., Kim, J. Y., Lee, D. K., Kim, D. E., Lee, J. \& Ko, M. J. Highly efficient perovskite solar cells based on mechanically durable molybdenum cathode. Nano Energy 17, 131-139 (2015)

23 Park, M., Kim, H. J., Jeong, I., Lee, J., Lee, H., Son, H. J., Kim, D. E. \& Ko, M. J. Mechanically recoverable and highly efficient perovskite solar cells: investigation of 
intrinsic flexibility of organic-inorganic perovskite. Adv. Energy Mater. 5, 1501406 (2015).

24 Kim, R. H., Kim, H. J., Bae, I., Hwang, S. K., Velusamy, D. B., Cho, S. M., Takaishi, K., Muto, T., Hashizume, D., Uchiyama, M., André, P., Mathevet, F., Heinrich, B., Aoyama, T., Kim, D. E., Lee, H., Ribierre, J. C. \& Park, C. Non-volatile organic memory with submillimetre bending radius. Nat. Commun. 5, 3583 (2014).

25 Sun, Y., Gates, B., Mayers, B. \& Xia, Y. Crystalline silver nanowires by soft solution processing. Nano Lett. 2, 165-168 (2002).

26 Sun, Y., Ren, Y., Liu, Y., Wen, J., Okasinski, J. S. \& Miller, D. J. Ambient-stable tetragonal phase in silver nanostructures. Nat. Commun. 3, 971 (2012)

27 Sorel, S., Bellet, D. \& Coleman, J. N. Relationship between material properties and transparent heater performance for both bulk-like and percolative nanostructured networks. ACS Nano 8, 4805-4814 (2014).

$28 \mathrm{Li}$, J., Liang, J., Jian, X., Hu, W., Li, J. \& Pei, Q. A flexible and transparent thin film heater based on a silver nanowire/heat-resistant polymer composite. Macromol. Mater. Eng. 299, 1403-1409 (2014).

29 You, B., Ju, B. K. \& Kim, J. W. Photoresist-assisted fabrication of thermally and mechanically stable silver nanowire-based transparent heaters. Sens. Actuators A Phys. 250, 123-128 (2016).

30 Kim, T. Y., Kim, Y. W., Lee, H. S., Kim, H., Yang, W. S. \& Suh, K. S. Uniformly interconnected silver-nanowire networks for transparent film heaters. Adv. Funct. Mater. 23, 1250-1255 (2013).

31 Wang, P. H., Chen, S. P., Su, C. H. \& Liao, Y. C. Direct printed silver nanowire thin film patterns for flexible transparent heaters with temperature gradients. RSC Adv. 5, 98412 (2015).

32 Cheong, H. G., Kim, J. H., Song, J. H., Jeong, U. \& Park, J. W. Highly flexible transparent thin film heaters based on silver nanowires and aluminum zinc oxides. Thin Solid Films 589, 633-641 (2015).

33 Lee, J., Woo, J. Y., Kim, J. T., Lee, B. Y. \& Han, C. S. Synergistically enhanced stability of highly flexible silver nanowire/carbon nanotube hybrid transparent electrodes by plasmonic welding. ACS Appl. Mater. Interfaces 6, 10974-10980 (2014).
34 Lai, Y. T. \& Tai, N. H. One-step process for high-performance, adhesive, flexible transparent conductive films based on p-type reduced graphene oxides and silver nanowires. ACS Appl. Mater. Interfaces 7, 18553-18559 (2015).

35 Berman, D., Erdemir, A. \& Sumant, A. V. Graphene: a new emerging lubricant. Mater. Today 17, 31-42 (2014).

36 Penkov, O., Kim, H. J., Kim, H. J. \& Kim, D. E. Tribology of graphene: a review. Int. J. Precis. Eng. Manuf. 15, 577-585 (2014).

$37 \mathrm{Ou}$, J., Wang, J., Liu, S., Mu, B., Ren, J., Wang, H. \& Yang, S. Tribology study of reduced graphene oxide sheets on silicon substrate synthesized via covalent assembly. Langmuir 26, 15830-15836 (2010).

38 Mungse, H. P. \& Khatri, O. P. Chemically functionalized reduced graphene oxide as a novel material for reduction of friction and wear. J. Phys. Chem. C 118, 14394-14402 (2014).

$39 \mathrm{Kim}$, D. E., Kim, C. L. \& Kim, H. J. A novel approach to wear reduction of microcomponents by synthesis of carbon nanotube-silver composite coating. CIRP Ann. Manuf. Technol. 60, 599-602 (2011).

$40 \mathrm{Kim}, \mathrm{C}$. L. \& Kim, D. E. Self-healing characteristics of collagen coatings with respect to surface abrasion. Sci. Rep. 6, 20563 (2016).

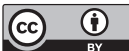

This work is licensed under a Creative Commons Attribution 4.0 International License. The images or other third party material in this article are included in the article's Creative Commons license, unless indicated otherwise in the credit line; if the material is not included under the Creative Commons license, users will need to obtain permission from the license holder to reproduce the material. To view a copy of this license, visit http:// creativecommons.org/licenses/by/4.0/

(C) The Author(s) 2017

Supplementary Information accompanies the paper on the NPG Asia Materials website (http://www.nature.com/am) 